\title{
Dynamic observations of dislocation/grain-boundary interactions in ice
}

\author{
I. Baker, ${ }^{1}$ F. Liu, ${ }^{1}$ K. Jia, ${ }^{1}$ X. Hu, ${ }^{1}$ D. Cullen, ${ }^{1}$ M. Dudley ${ }^{2}$ \\ ${ }^{1}$ Thayer School of Engineering, Dartmouth College, Hanover, NH 03755, U.S.A. \\ ${ }^{2}$ Department of Materials Science and Engineering, State University of New York at Stony Brook, Stony Brook, NY 11794, U.S.A.
}

\begin{abstract}
Dislocation/grain-boundary (GB) interactions have been studied in situ in polycrystalline ice using synchrotron X-ray topography in the temperature range $0^{\circ}$ to $-15^{\circ} \mathrm{C}$. GBs were observed to act both as sources of lattice dislocations and as strong obstacles to dislocation motion. Dislocations were observed to form pile-ups at GBs upon loading. Generally, the basal slip system with the highest Schmid factor was found to be the most active, and dislocations were emitted from GB facets as semi-hexagonal loops in order to relieve the stress build-up from GB sliding. When the relative orientation of two adjacent grains and the orientation of the GB between them with respect to the loading direction discouraged GB sliding, thus suppressing dislocation nucleation at the GB, dislocations originating in one grain piled up at the GB and led to slip transmission through the GB. The latter geometrical arrangement is rarely encountered, suggesting that slip transmission through grain boundaries in ice is a rare event. When basal slip was suppressed, i.e. when the loading direction lay in the basal plane, slip occurred by the glide of a fast edge segment on non-basal planes.
\end{abstract}

\section{INTRODUCTION}

An understanding of both the structure and dynamics of the dislocations in a material is fundamental to an understanding of its mechanical properties. X-ray topography, which produces two-dimensional projections of the three-dimensional defect structure of a material, has proven to be the most useful technique for examining dislocations in ice.

Conventional X-ray topography, which involves scanning the ice crystal with a weak monochromatic X-ray beam, was first applied to the observation of dislocations in ice by Hayes and Webb (1965), and has made useful contributions ever since. Slip dislocations in ice have been shown both to have a $1 / 3\langle 2 \overline{1} \overline{1} 0\rangle$ Burgers vector and to slip either on the basal plane or on non-basal planes, such as the prismatic planes (Higashi, 1988). One disadvantage with conventional $\mathrm{X}$-ray topography is that only one diffraction condition is imaged at a time. However, the key problem is that exposure times are long, typically $>1 \mathrm{~h}$. This means that for observations above $-30^{\circ} \mathrm{C}$ substantial recovery takes place. This method is therefore unable to reveal the true dislocation behavior in ice under stress, as indicated by the topographs that show blurred images and/or curved dislocations and the formation of small-angle grain boundaries after deformation and load removal. The straight dislocations that lie in Peierls valleys during deformation (see below) are rarely seen due to recovery. Thus, images from conventional X-ray topography are not easily interpretable due to these recovery effects.

Synchrotron X-ray topography is a simpler technique than conventional X-ray topography. The key advantage is that the high-intensity X-rays allow exposure times of $\sim 2 \mathrm{~s}$, enabling dynamic experiments and unrecovered dislocation structures to be observed. The broad spectral range of the area-filling beam also means that several grains in largegrained polycrystals can be imaged simultaneously.

R.W. Whitworth's group first used synchrotron X-ray topography to observe the dynamic behavior of dislocations, and the most important contributions on single-crystal ice are from his group (Ahmad and others, 1986, 1987, 1992; Ahmad and Whitworth, 1988; Shearwood and Whitworth, 1989, 1991, 1992, 1993). It was shown that under stress screw and $60^{\circ}$ basal dislocations predominate in ice, rather than the curved dislocations typically observed by conventional X-ray topography. The motion of the edge segment on the non-basal plane was shown to be faster than that of the screw dislocations on the basal plane. This plays an important role in deformation because it provides the principal mechanism for the generation and multiplication of the dislocations that subsequently move on the basal planes and produce macroscopic basal slip. In addition, dynamic straining experiments (Hondoh and others, 1990; Shearwood and Whitworth, 1991, 1992; Hu and others, 1995) have been used to disclose dislocation velocities as a function of both stress and temperature. Thus, the deformation mechanisms of single-crystal ice are now fairly well established.

Most early progress related to the deformation of ice containing a large-angle grain boundary (GB) has been made by A. Higashi and T. Hondoh's group (Higashi, 1978; Hondoh and Higashi, 1978, 1979, 1983; Hondoh, 1986, 1988, 1992) using the conventional Lang method to examine bicrystals. Upon stressing ice samples, strain fields, a facet structure and both dislocation generation and absorption were observed at high-angle GBs. The strained (darkened) areas at the GBs were also observed to extend into the interior with further loading. The structure and behavior of the large-angle GBs were interpreted using a near coincidence-site lattice theory. 


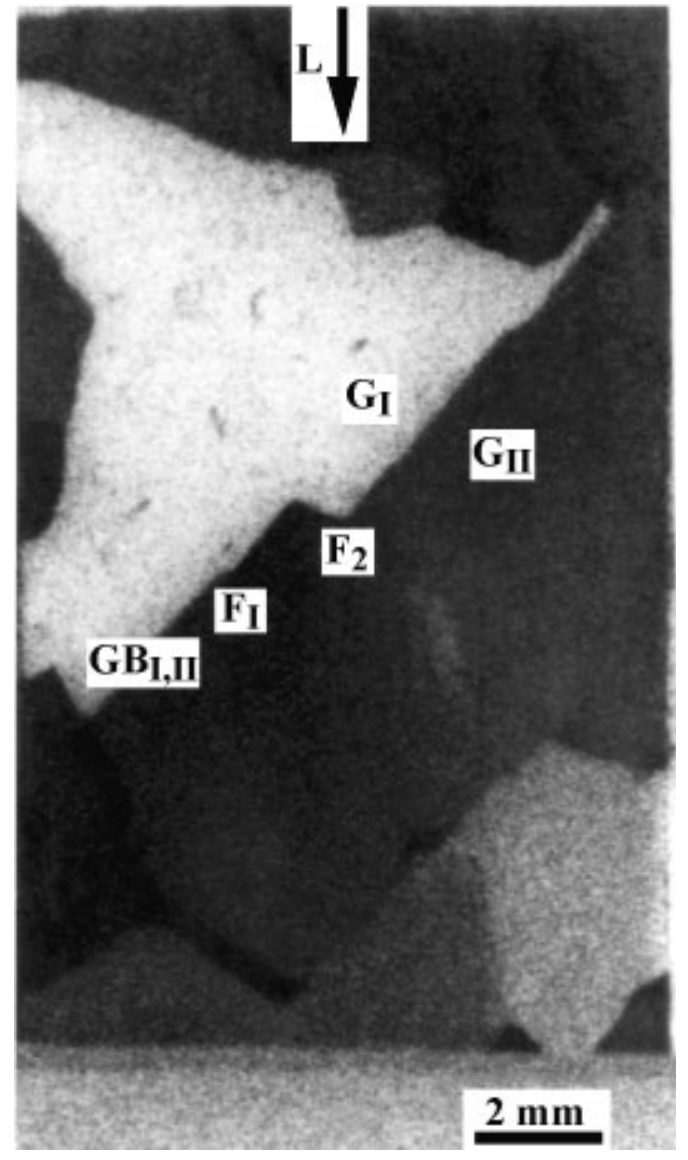

Fig. 1. An optical micrograph of polycrystalline ice specimens observed between cross-polarized sheets. Note the large GB facet.
The formation of several small-angle GBs in the vicinity of the original GB after removing the load was also reported, indicating the recovered nature of the structures observed using conventional X-ray topography. The work also could not identify the Burgers vectors of dislocations in polycrystalline ice because of the difficulty involved in rotating the specimens in the particular apparatus used. Therefore, the response of the second grain, to which strain was transferred, was not clear; dislocations which had reacted with the GB may not have been visible because of the specific diffraction condition used: that is, one diffraction spot cannot provide complete information about dislocation structures.

Synchrotron X-ray topography was first applied to polycrystalline ice by the principal author's group (Liu and others, 1992a). The purpose of this paper is to outline dynamic synchrotron X-ray topography observations of dislocation behavior in polycrystalline ice under creep conditions.

\section{EXPERIMENTAL}

High-quality, high-purity, columnar-grained ice was grown in the Ice Research Laboratory, Thayer School of Engineering, Dartmouth College. On examining ice pucks between crosspolarized sheets, it was found that GB facets were common (see Fig. 1). Thin $(25 \mathrm{~mm}$ high $\times 12 \mathrm{~mm}$ wide $\times 2 \mathrm{~mm}$ thick $)$ polycrystalline specimens were made from the ice pucks by sawing and planing; details are given elsewhere (Liu and others, 1992a). The specimens were sealed in Melinex chambers (Liu and Baker, 1993) and transported to the National

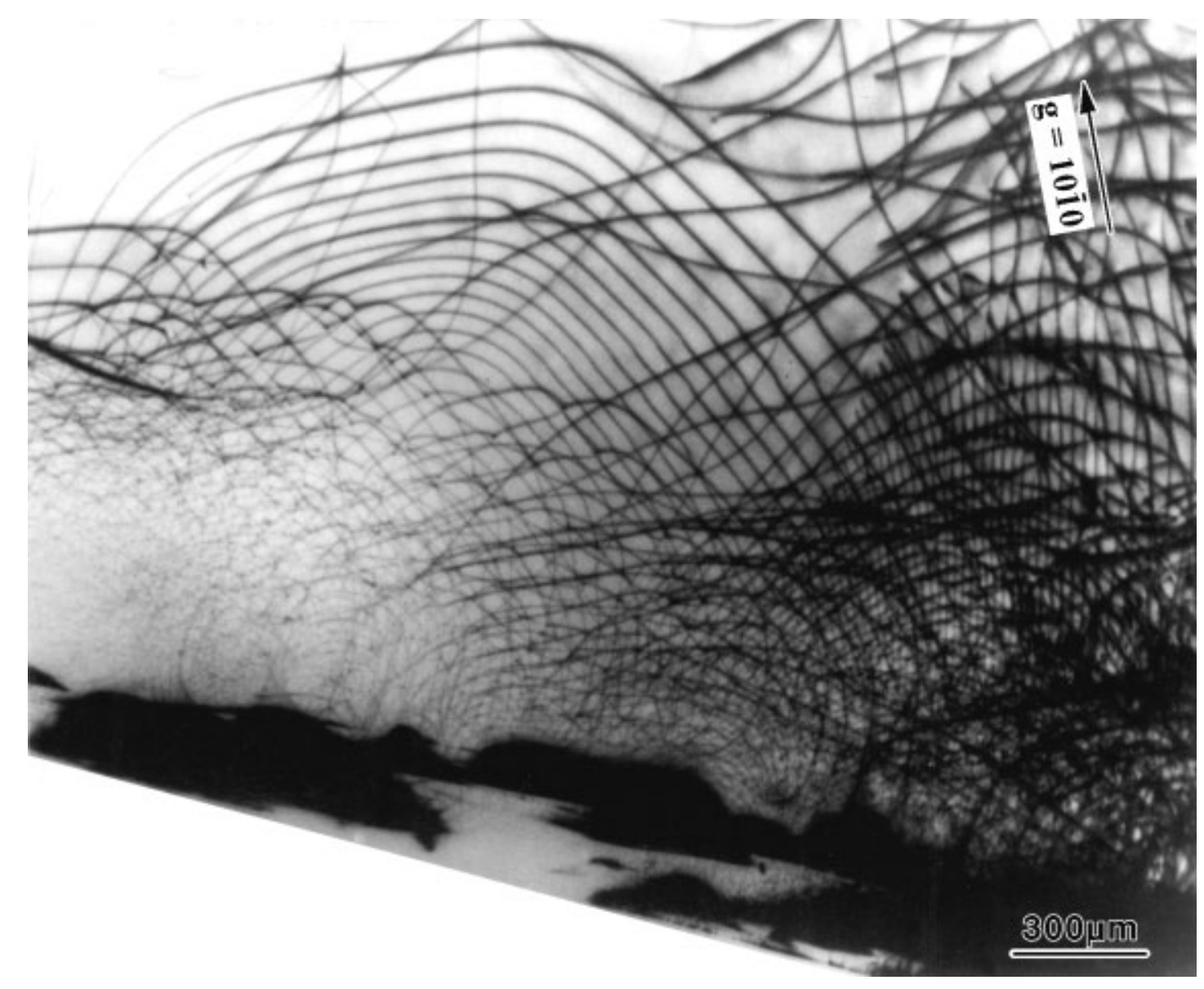

Fig. 2. After loading specimen 1, numerous semi-hexagonal loops have been emitted from the $G B$ at $\sim 10^{\circ} \mathrm{C}$. The black areas on the $G B$ are due to stress concentrations there. The diffraction vector, $g=10 \overline{1} 0$, is indicated. 
Synchrotron Light Source (NSLS), Brookhaven National Laboratory, NY, without either significant specimen sublimation or frost formation.

In situ deformation studies were performed on the ice using a specially built testing jig assembled with a cryostat (Liu and Baker, 1993) at NSLS. The desired loads and load durations were chosen based on the dislocation mobility data of Shearwood and Whitworth (1991). In the experiments reported here, the temperatures used were in the range $0^{\circ}$ to $-15^{\circ} \mathrm{C}$. Topographs (Laue patterns) were produced by allowing a highly collimated beam of white X-rays (with the NSLS operating at $2.5 \mathrm{GeV}$ and $\sim 180 \mathrm{~mA}$ ) to fall on the specimen; details are given elsewhere (Liu and Baker, 1994). Several diffraction spots (from each grain) were recorded simultaneously on a single X-ray film using exposure times of $\sim 2 \mathrm{~s}$. The microstructural evolution during loading was recorded as long sequences of topographs; only selected topographs are reproduced here.

Over 25 specimens were studied, but only four specimens, which show all the possible features of the deformation of polycrystalline ice, are discussed here. Specimen 1 was cut with no particular preference given to the grain boundary or grains with respect to the loading-direction orientation. Specimens 2 and 3 were cut from the same piece of ice such that each contained the same four grains and the same long GB in the center of the specimen could be studied in each. In specimen 2, this GB makes a $45^{\circ}$ angle with the loading direction (the specimen's vertical axis), a fairly general configuration. In specimen 3 , the loading direction lies in the GB plane, thus suppressing grain-boundary sliding. Specimen 4 was cut so that for the grain of interest the loading direction was in the basal plane. Thus, the resolved shear stress on the basal plane from the applied far-field stress was zero.

\section{RESULTS AND DISGUSSION}

For polycrystalline ice, three different geometrical situations are possible when loading ice uniaxially under creep conditions (Liu and others, 1992b, 1995; Jia and others, 1996). First, for grains and grain boundaries which are not oriented in any special relationships with the loading direction, screw and $60^{\circ}$ basal $1 / 3\langle 2 \overline{1} \overline{1} 0\rangle$ dislocations are emitted from grainboundary facets as semi-hexagonal dislocation loops (see specimen 1 in Fig. 2). The basal slip system with the highest Schmid factor appears to be the most active in ice. The dislocations are emitted from the GB in order to relax stress concentrations at the grain-boundary facets, which arise from grain-boundary sliding (specimen 2 in Fig. 3). Stress concentrations are evident from the darkening observed around the facets in the topographs. Neither dislocation absorption at, nor dislocation pile-up in front of, a GB is necessary for dislocations to nucleate in adjacent grains. Only the GBs appear to act as sources of dislocations. In other words, dislocation nucleation mechanisms which operate in the grain interior, as observed in single crystals (Ahmad and others, 1986, 1987; Shearwood and Whitworth, 1993), are not important. Eventually, the dislocations which have been emitted traverse the grain and pile up at the opposite GB (see Fig. 3). Thus, the GBs act as strong obstacles to dislocation motion.

The second situation occurs when grain-boundary sliding is suppressed. In that case, there are no stress build-ups at the GBs and consequent dislocation emissions from grain-
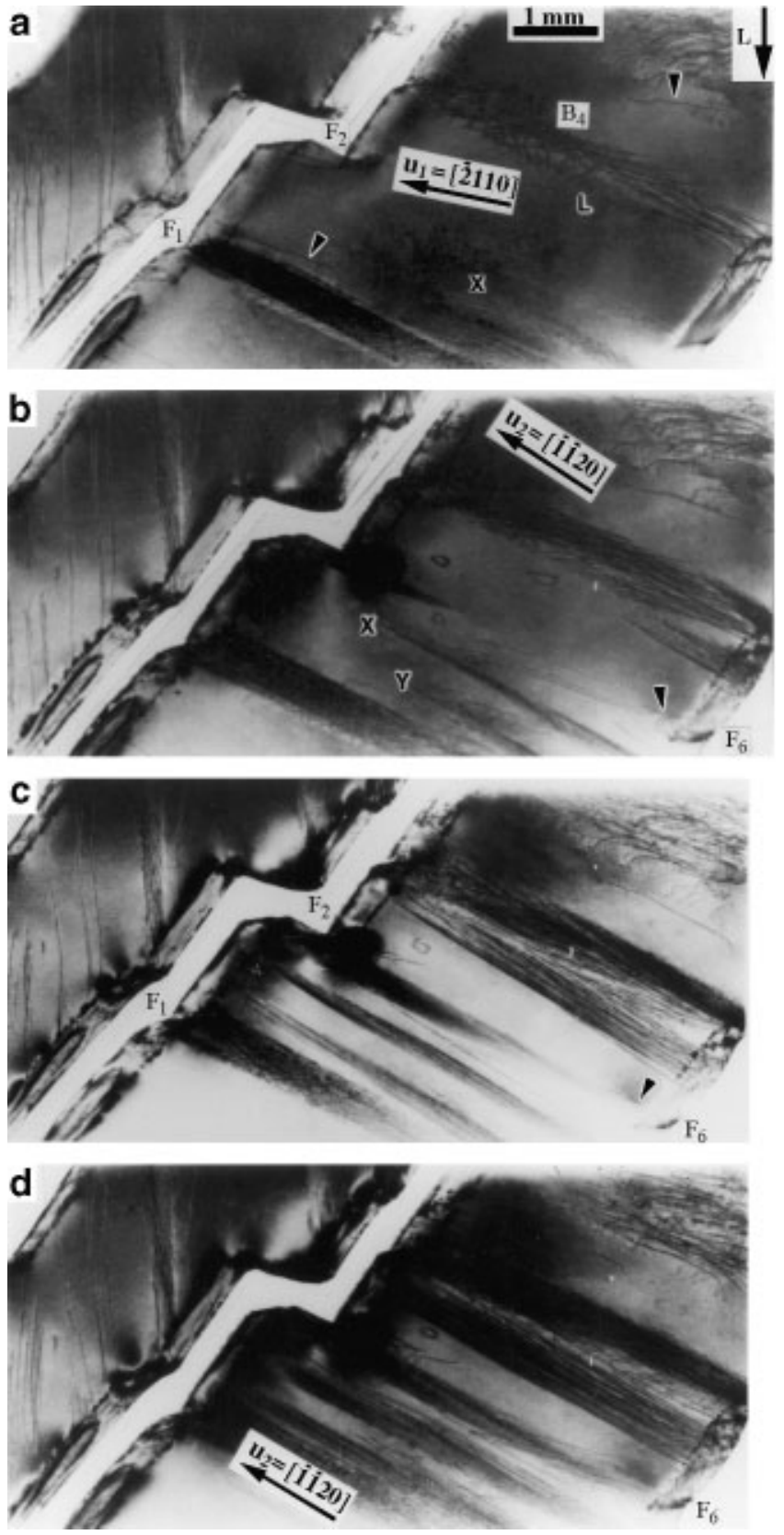

Fig. 3. X-ray topographs taken at $-6.0^{\circ} \mathrm{C}$ of specimen 2 at increasing load (the load direction is marked as L) showing basal dislocations which have been emitted at GB facets into two adjacent grains in order to accommodate GB sliding. Note the dislocations in the slip bands labeled $x$ which nucleated at the GB facet in the lower right. These traversed the righthand grain progressively further in $(b-d)$ and eventually pile up at the opposite GB. The load was (a) $0.1 \mathrm{MPa}$ for $5 \mathrm{~min}$; ( $b$ ) $0.2 \mathrm{MPa}$ for $10 \mathrm{~min}$; (c) $0.2 \mathrm{MPa}$ for a further $20 \mathrm{~min}$; and (d) $0.2 \mathrm{MPa}$ for a further $25 \mathrm{~min}$.

boundary facets. Then, under the correct and somewhat exacting geometrical conditions, i.e. some alignment of the basal slip planes in the two grains, slip transmission can occur. The topographs of specimen 3 in Figure 4, taken at increasingly higher load applied to the specimen, show this situation. Even the lowest applied load (0.16 MPa) caused darkening at the GB, indicative of internal stresses. As the load continued to increase, the extent of the darkened area also increased, indicating the continuing stress build-up there. During initial loading there was some movement of 

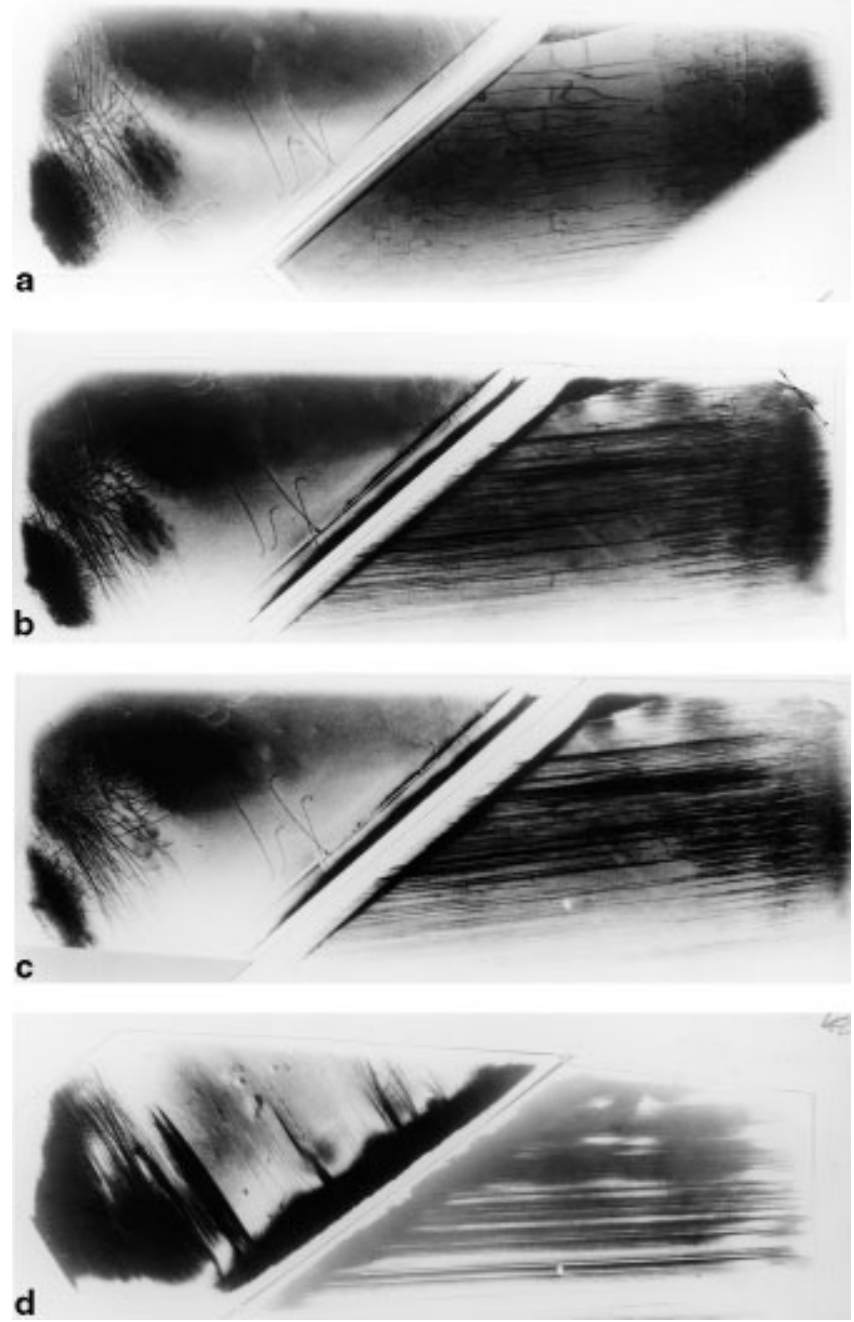

Fig. 4. Topographs taken at $-12^{\circ} \mathrm{C}$ showing (1010) images of two grains in specimen 3 in which the loading direction lies in the boundary plane between them. Note that the boundary does not contain any facets and that the basal plane in the righthand grain is viewed almost edge on. The dislocations impinging on the $G B$ in the righthand grain eventually lead to slip in the lefthand grain. (a) After loading at $0.16 \mathrm{MPa}$ for $38 \mathrm{~min}$; (b) $0.23 \mathrm{MPa}$ for $10 \mathrm{~min}$ and $0.31 \mathrm{MPa}$ for $5 \mathrm{~min}$; (c) $0.35 \mathrm{MPa}$ for $10 \mathrm{~min}$; (d) $0.29 \mathrm{MPa}$ for $30 \mathrm{~min}$ and $0.33 \mathrm{MPa}$ for $30 \mathrm{~min}$.

dislocations in the lefthand grain, but in the righthand grain there was much more significant movement of the dislocations towards the GB, and they can clearly be seen to intersect the GB. The straight basal dislocations in the righthand grain appear as dark lines parallel to the trace of the basal plane. Eventually, as higher loads were reached, new dislocations nucleated at the GB and moved into the lefthand grain (Fig. 4). The results clearly showed that slip transmission occurred through a GB in ice, i.e. dislocation impingement on one side of a GB led to dislocations being emitted from the other side of the GB. Due to the low resolution of $\mathrm{X}$-ray topography, it was not possible to distinguish between dislocation transmission through the GB and nucleation of dislocations at the GB that arose from the stress-concentrating effect of the impinging dislocations.

The third situation occurs when basal slip is suppressed, i.e. when the resolved shear stress on basal slip systems from the applied far-field stress is close to zero. This occurs when

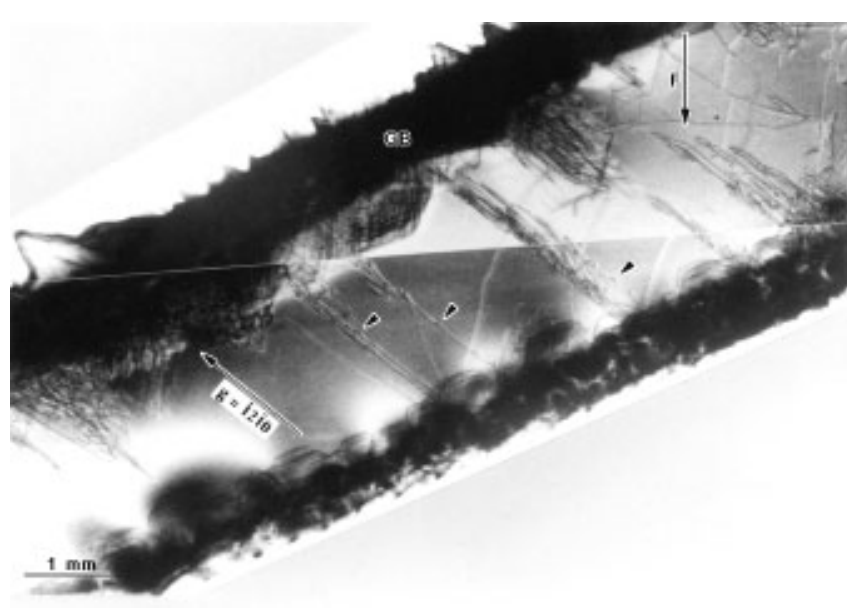

Fig. 5. X-ray topograph at $-6^{\circ} \mathrm{C}$ showing the generation of both semi-hexagonal basal dislocation loops and non-basal edge segments at GBs in specimen 4 . The GBs are almost black due to the large local stress concentrations. Interestingly, semihexagonal basal dislocation loops have also been nucleated at the GBs even though the resolved shear stress from the far-field applied stress is close to zero.

the loading direction is nearly perpendicular to either the basal plane or the basal plane normal. When the loading direction is perpendicular to the basal plane normal, nonbasal $1 / 3\langle 2 \overline{1} \overline{1} 0\rangle$ dislocations can be emitted from GB facets. Figure 5 shows this situation for specimen 4, where slip occurs by the glide of short fast edge segments on non-basal planes, which trail screw segments behind them. Eventually, at higher strains the local stresses generated at the GB lead to the nucleation of semi-hexagonal dislocation loops (see Fig. 5). Note that the basal loops do not glide far into the grain since the resolved shear stress on the basal plane from the far-field stress is close to zero.

It is worth noting that the latter two situations are geometrically unusual and would occur very infrequently in naturally deforming polycrystalline ice. In particular, the slip transmission event studied was special for several reasons. First, GB sliding was suppressed. Second, there were no observable facets on the GB. Both of these prevent dislocation nucleation at the boundary as described in the first situation above. Third, the angle between the basal planes on either side of the GB was small $\left(11^{\circ}\right)$, favoring slip transmission from one grain to the next. Thus, slip transmission occurred in a rather special situation. Note that in experiments on over 25 boundaries in the same temperature and stress ranges a slip transmission event was observed only once.

\section{GONGLUSIONS}

Dynamic-loading in situ X-ray topography under creep conditions has been used to examine dislocation/GB interactions in polycrystalline ice. The interaction mechanisms depend strongly on the orientation of the basal slip systems relative to the loading direction and the GB plane. Dislocation nucleation always occurred at the GBs and not within the grain interiors. Three different situations were observed. First, for grains and GBs that do not possess any special relationships with the loading direction, $1 / 3\langle 2 \overline{1} \overline{1} 0\rangle$ dislocations are emitted on the basal plane as semi-hexagonal loops of screw and $60^{\circ}$ dislocations. This emission occurs at GB facets in 
order to accommodate GB sliding, which leads to stress concentrations at the facets. In this case, the basal slip system with the highest Schmid factor is the most active. The second situation occurs when GB sliding is suppressed so that there are no stress build-ups at GBs and dislocation emission from GB facets does not occur. Then, under the correct geometrical conditions, i.e. some alignment of the basal slip planes, slip transmission from one grain to the next can occur. Third, when basal slip is suppressed, non-basal $1 / 3\langle 2 \overline{1} \overline{1} 0\rangle$ dislocations can be emitted from GB facets. Dislocation pile-ups at GBs were observed in several instances.

\section{ACKNOWLEDGEMENTS}

This research was supported by grant OPP-9526454 from the U.S. National Science Foundation and grant DAAH04-96-1-0041 from the U.S. Army Research Office.

\section{REFERENGES}

Ahmad, S. and R.W. Whitworth. 1988. Dislocation motion in ice: a study by synchrotron X-ray topography. Philos. Mag. A, 57(5), 749-766.

Ahmad, S., M. Ohtomo and R.W. Whitworth. 1986. Observation of a dislocation source in ice by synchrotron radiation X-ray topography. Nature, 319(6055), 659-660.

Ahmad, S., M. Ohtomo and R.W. Whitworth. 1987. A study of dislocation glide in ice by synchrotron radiation X-ray topography. 7. Phys. (Paris), 48, Colloq. C1, 175-181. (Supplément au 3.)

Ahmad, S., C. Shearwood and R.W. Whitworth. 1992. Dislocation multiplication mechanisms in ice. In Maeno, N. and T. Hondoh, eds. Proceedings of the International Symposium on the Physics and Chemistry of Ice, Sapporo, Fapan. Sapporo, Hokkaido University Press, 492-496.

Hayes, C. E. and W.W. Webb. 1965. Dislocations in ice. Science, 147, 44-45.

Higashi, A. 1978. Structure and behaviour of grain boundaries in polycrystalline ice. 7. Glaciol., 21 (85), 589-605.

Higashi, A. 1988. Lattice defects in ice crystals: X-ray topographic observations. Sapporo, Japan, Hokkaido University Press.

Hondoh, T. 1986. In-situ X-ray topographic observations of stress concentrations at a serrated grain boundary in ice under deformation, grain boundary structure and related phenomena. Trans. Jpn. Inst. Met., Sup- plement 27, 723-729. (Proceedings of JIMIS-4.)

Hondoh, T. 1988. Observations of large-angle grain boundaries in ice crystals. In Higashi, A., ed. Lattice defects in ice crystals; X-ray topographic observations. Sapporo, Hokkaido University Press, 129-146.

Hondoh, T. 1992. Glide and climb processes of dislocations in ice. In Maeno, N. and T. Hondoh, eds. Proceedings of the International Symposium on the Physics and Chemistry of Ice, Sapporo, Japan. Sapporo, Hokkaido University Press, 481-487.

Hondoh, T. and A. Higashi. 1978. X-ray diffraction topographic observations of the large-angle grain boundary in ice under deformation. f. Glaciol., $21(85), 629-638$.

Hondoh, T. and A. Higashi. 1979. Anisotropy of migration and faceting of large-angle grain boundaries in ice bicrystals. Philos. Mag. A, 39(2), 137-149.

Hondoh, T. and A. Higashi. 1983. Generation and absorption of dislocations at large-angle grain boundaries in deformed ice crystals. F. Phys. Chem., 87(21), 4044-4050.

Hondoh, T., H. Iwamatsu and S. Mae. 1990. Dislocation mobility for nonbasal glide in ice measured by in situ X-ray topography. Philos. Mag. A, 62(1), 89-102.

Hu, X., K. Jia, P. Liu, I. Baker and D. Black. 1995. Dislocation mobility in HCldoped ice. Materials Research Society Symposium Proceedings, 375, 287-292.

Jia, K., I. Baker, F. Liu and M. Dudley. 1996. Observation of slip transmission through a grain boundary in ice. F. Mater. Sci., 31 (9), 2373-2378.

Liu, F. and I. Baker. 1993. A compression jig for X-ray topography of ice. Measurement Science and Technology, 4(3), 416-421.

Liu, F. and I. Baker. 1994. Synchrotron X-ray topographic studies of grain boundaries. Microscopy Society of America Bulletin, 24(1), 351-358.

Liu, F., I. Baker, G. Yao and M. Dudley. 1992a. Dislocations and grain boundaries in polycrystalline ice: a preliminary study by synchrotron X-ray topography. 7. Mater. Sci., 27 (10), 2719-2725.

Liu, F., I. Baker, G. Yao and M. Dudley. 1992b. Dynamic observations of dislocation sources at grain boundaries in ice. Philos. Mag. Lett., 65(5), 279-281.

Liu, F. P., I. Baker and M. Dudley. 1995. Dislocation/grain boundary interactions in ice crystals. Philos. Mag. A, 71 (1), 15-42.

Shearwood, C. and R. W. Whitworth. 1989. X-ray topographic observations of edge dislocation glide on non-basal planes in ice. f. Glaciol., 35(120), 281-283.

Shearwood, C. and R.W. Whitworth. 1991. The velocity of dislocations in ice. Philos. Mag. A, 64(2), 289-302.

Shearwood, C. and R.W. Whitworth. 1992. The velocity of dislocations in crystals of HCl-doped ice. Philos. Mag. A, 65(1), 85-89.

Shearwood, C. and R.W. Whitworth. 1993. Novel processes of dislocation multiplication observed in ice. Acta Metall. Materialia, 41 (1), 205-210. 